\title{
On the Second-Order Quantum $(p, q)$-Difference Equations with Separated Boundary Conditions
}

\author{
Chanon Promsakon, ${ }^{1}$ Nattapong Kamsrisuk, ${ }^{1}$ \\ Sotiris K. Ntouyas $\mathbb{D}^{2,3}$ and Jessada Tariboon $\mathbb{D}^{1}$ \\ ${ }^{1}$ Intelligent and Nonlinear Dynamic Innovations Research Center, Department of Mathematics, Faculty of Applied Science, \\ King Mongkut's University of Technology North Bangkok, Bangkok 10800, Thailand \\ ${ }^{2}$ Department of Mathematics, University of Ioannina, 45110 Ioannina, Greece \\ ${ }^{3}$ Nonlinear Analysis and Applied Mathematics (NAAM)-Research Group, Department of Mathematics, Faculty of Science, \\ King Abdulaziz University, P.O. Box 80203, Jeddah 21589, Saudi Arabia \\ Correspondence should be addressed to Jessada Tariboon; jessada.t@sci.kmutnb.ac.th
}

Received 19 August 2018; Accepted 12 November 2018; Published 2 December 2018

Academic Editor: Antonio Scarfone

Copyright (C) 2018 Chanon Promsakon et al. This is an open access article distributed under the Creative Commons Attribution License, which permits unrestricted use, distribution, and reproduction in any medium, provided the original work is properly cited.

In this paper, we investigate the existence and uniqueness of solutions for a boundary value problem for second-order quantum $(p, q)$-difference equations with separated boundary conditions, by using classical fixed point theorems. Examples illustrating the main results are also presented.

\section{Introduction}

Quantum calculus or $q$-calculus is known as the study of calculus without limits. The study of $q$-calculus was initiated by Euler on studying infinite series. In 1910, Jackson $[1,2]$ was the first one, who establised the $q$-derivative or $q$-difference operator for a function $f$ on $[0, \infty)$ by

$$
\begin{aligned}
D_{q} f(t) & =\frac{f(t)-f(q t)}{(1-q) t}, \quad t \neq 0, \\
\text { and } D_{q} f(0) & =\lim _{t \rightarrow 0} D_{q} f(t), \quad t=0,
\end{aligned}
$$

and gave its properties. The $q$-integral of a function $f$ on $[0, \infty)$ is defined by

$$
\int_{0}^{t} f(s) d_{q} s=(1-q) t \sum_{n=0}^{\infty} q^{n} f\left(q^{n} t\right)
$$

provided that infinite series converges. The general theory of linear quantum difference equations was published in 1912 by Carmichael [3]. Details of its basic notions, results, and methods can be found in the text [4]. For other papers on the subject, see [5-7]. In recent years, the topic has been attracting the attention of several researchers and a variety of new results can be found in [8-13] and the references therein. In addition, the classical quantum calculus was generalized to $q_{k}$-calculus by Tariboon and Ntouyas [14]. For details, we refer to the recent monograph [15].

There are some applications of $q$-calculus and difference equations to molecular problems in physics. In 1967, Finkelstein [16] studied behaviors of hydrogen atoms by using Schrödinger equation and $q$-calculus. In [17], the author investigated the $q$-field theory. The $q$-Coulomb problem and $q$-hydrogen atom were studied by [18-21]. In addition, YangMills theories and also $q$-Yang-Mills equation were developed by [22-24]. The theory of quantum group applied to vibration and rotation molecules with $q$-algebra and $q$-Heisemberg algebra technique was established in [25-27]. The subject of elementary particle physics and chemical physics using $q$-calculus was investigated in [28-31]. The string theory involving $q$-calculus was studied in [32].

The subject of $(p, q)$-calculus first appeared in quantum algebras which contained two quantum numbers $p$ and 
$q$ introduced by [33]. For some recent results see [3441] and the references cited therein. Recently in [42], the authors initiated the study on boundary value problems for $(p, q)$-difference equations by investigating the existence and uniqueness of solution for first-order quantum $(p, q)$ difference equation subject to a nonlocal condition of the form

$$
\begin{aligned}
D_{p, q} x(t) & =f(t, x(p t)), \quad t \in\left[0, \frac{T}{p}\right], \\
x(0) & =\alpha x(T)+\sum_{i=1}^{m} \beta_{i} \int_{0}^{\eta_{i}} x(s) d_{p_{i}, q_{i}} s
\end{aligned}
$$

where $0<q<p \leq 1,0<q_{i}<p_{i} \leq 1, i=1,2, \ldots, m$, are quantum numbers, $D_{p, q}$ is $(p, q)$-difference operator, $f \in$ $C([0, T / p] \times \mathbb{R}, \mathbb{R}), T>0, \alpha, \beta_{i}, i=1,2, \ldots, m$, are given constants, and the points $\eta_{i} \in\left[0, p_{i} T\right], i=1,2, \ldots, m$. They proved existence and uniqueness results for problem (3)-(4) by using the classical fixed point theorems, such as Banach's fixed point theorem, Boyd and Wong fixed point theorem for nonlinear contractions, and Leray-Schauder nonlinear alternative.

In this paper, we continue the study on $(p, q)$ boundary value problems, by considering the following second-order quantum $(p, q)$-difference equation with separated boundary conditions

$$
\begin{aligned}
& D_{p, q}^{2} x(t)=f\left(t, x\left(p^{2} t\right)\right), \\
& t \in\left[0, \frac{T}{p^{2}}\right], \\
& \alpha_{1} x(0)+\alpha_{2} D_{p, q} x(0)=\alpha_{3}, \\
& \beta_{1} x(T)+\beta_{2} D_{p, q} x\left(\frac{T}{p}\right)=\beta_{3},
\end{aligned}
$$

where $0<q<p \leq 1$ are two given quantum numbers, $D_{p, q}^{2}$ is the second-order $(p, q)$-difference operator, and $f \in$ $C\left(\left[0, T / p^{2}\right] \times \mathbb{R}, \mathbb{R}\right), T>0, \alpha_{i}, \beta_{i}, i=1,2,3$, are given real constants. Some new existence results are established by using Schaefer fixed point theorem, Krasnoselskii's fixed point theorem, and Lelay-Schauder's nonlinear alternative. In addition the uniqueness of solutions is established via Banach contraction mapping principle.

The paper is organized as follows: In Section 2, we recall some definitions and basic facts from $(p, q)$-calculus. The main existence and uniqueness results are given in Section 3. Examples illustrating the obtained results are presented in Section 4.

\section{Preliminaries}

In this section, we recall some basic concepts of $(p, q)$ calculus (see $[41])$. The $(p, q)$-number is defined by

$$
[n]_{p, q}=\frac{p^{n}-q^{n}}{p-q}
$$

where $0<q<p \leq 1$. For each $k, n \in \mathbb{N}, n \geq k \geq 0$, the $(p, q)$-factorial and $(p, q)$-binomial are defined by

$$
[n]_{p, q} !=\prod_{k=1}^{n}[k]_{p, q}, \quad n \geq 1,[0]_{p, q} !=1,
$$

and

$$
\left[\begin{array}{l}
n \\
k
\end{array}\right]_{p, q}=\frac{[n]_{p, q} !}{[n-k]_{p, q} ![k]_{p, q} !}
$$

respectively.

Let $f:[0, T] \longrightarrow \mathbb{R}$. The $(p, q)$-derivative of function $f$ is defined as

$$
D_{p, q} f(t)=\frac{f(p t)-f(q t)}{(p-q) t}, \quad t \neq 0,
$$

and $D_{p, q} f(0)=\lim _{t \rightarrow 0} D_{p, q} f(t)$. Observe that the function $D_{p, q} f(t)$ is defined on $[0, T / p]$ which is extended from $[0, T]$ of a function $f(t)$. In addition, we say that $f$ is $(p, q)$ differentiable on $[0, T / p]$ provided that $D_{p, q} f(t)$ exists for all $t \in[0, T / p]$.

Let a function $f:[0, T] \longrightarrow \mathbb{R}$. Then the $(p, q)$-integral of $f$ is defined by

$$
\int_{0}^{t} f(s) d_{p, q} s=(p-q) t \sum_{n=0}^{\infty} \frac{q^{n}}{p^{n+1}} f\left(\frac{q^{n}}{p^{n+1}} t\right),
$$

provided that the right hand side converges. Note that the domain of function $\int_{0}^{t} f(s) d_{p, q} s$ is $[0, p T]$ which shrinks from $[0, T]$ of a function $f(t)$ since $0<p \leq 1$.

In the following theorems we collect the basic properties of $(p, q)$-differentiation and $(p, q)$-integration, respectively. See [37].

Theorem 1 (see [37]). Suppose that $f, g:[0, T] \rightarrow \mathbb{R}$ are $(p, q)$-differentiable on $[0, T / p]$. Then and

(a) $f+g:[0, T] \longrightarrow \mathbb{R}$ is $(p, q)$-differentiable on $[0, T / p]$,

$$
D_{p, q}(f(t)+g(t))=D_{p, q} f(t)+D_{p, q} g(t) .
$$

(b) $\lambda f:[0, T] \longrightarrow \mathbb{R}$ is $(p, q)$-differentiable on $[0, T / p]$ for any constant $\lambda$, and

$$
D_{p, q}(\lambda f)(t)=\lambda D_{p, q} f(t) .
$$
and

(c) $f g:[0, T] \longrightarrow \mathbb{R}$ is $(p, q)$-differentiable on $[0, T / p]$,

$$
\begin{aligned}
& D_{p, q}(f g)(t)=f(p t) D_{p, q} g(t)+g(q t) D_{p, q} f(t) . \\
& D_{p, q}(f g)(t)=g(p t) D_{p, q} f(t)+f(q t) D_{p, q} g(t) .
\end{aligned}
$$
with

(d) If $g(t) \neq 0$, then $f / g$ is $(p, q)$-differentiable on $[0, T / p]$

$$
\begin{aligned}
& D_{p, q}\left(\frac{f}{g}\right)(t)=\frac{g(q t) D_{p, q} f(t)-f(q t) D_{p, q} g(t)}{g(p t) g(q t)} . \\
& D_{p, q}\left(\frac{f}{g}\right)(t)=\frac{g(p t) D_{p, q} f(t)-f(p t) D_{p, q} g(t)}{g(p t) g(q t)} .
\end{aligned}
$$


Theorem 2 (see [37]). Let $f, g:[0, T] \longrightarrow \mathbb{R}$ be continuous functions and constants $0<q<p \leq 1, a, b \in[0, T]$. The following formulas hold:

(a) The $(p, q)$-integration by parts is given by

$$
\begin{aligned}
& \int_{a}^{b} f(p t) D_{p, q} g(t) d_{p, q} t \\
& \quad=\left.f(t) g(t)\right|_{a} ^{b}-\int_{a}^{b} g(q t) D_{p, q} f(t) d_{p, q} t .
\end{aligned}
$$

(b) $D_{p, q} \int_{0}^{t} f(s) d_{p, q} s=f(t)$.

(c) $\int_{0}^{t} D_{p, q} f(s) d_{p, q} s=f(t)-f(0)$.

(d) $\int_{a}^{t} D_{p, q} f(s) d_{p, q} s=f(t)-f(a)$ where $a \in(0, t)$.

Theorem 3 (see [42]). Let a function $f:[0, T] \rightarrow \mathbb{R}$ and constants $0<q<p \leq 1$. Then for $t \in\left[0, p^{2} T\right]$, we have

$$
\int_{0}^{t} \int_{0}^{s} f(r) d_{p, q} r d_{p, q} s=\frac{1}{p} \int_{0}^{t}(t-q s) f\left(\frac{1}{p} s\right) d_{p, q} s .
$$

The second-order $(p, q)$-difference of a function $f$ : $[0, T] \longrightarrow \mathbb{R}$ can be expressed as

$$
\begin{aligned}
D_{p, q}^{2} f(t) & =D_{p, q}\left(D_{p, q} f\right)(t) \\
= & D_{p, q}\left(\frac{f(p t)-f(q t)}{(p-q) t}\right) \\
= & \frac{f\left(p^{2} t\right)-f(p q t)}{p(p-q)^{2} t^{2}}-\frac{f(p q t)-f\left(q^{2} t\right)}{q(p-q)^{2} t^{2}} \\
= & \frac{q f\left(p^{2} t\right)-q f(p q t)-p f(p q t)+p f\left(q^{2} t\right)}{p q(p-q)^{2} t^{2}} \\
= & \frac{q f\left(p^{2} t\right)-(p+q) f(p q t)+p f\left(q^{2} t\right)}{p q(p-q)^{2} t^{2}}, \\
& t \in\left[0, \frac{T}{p^{2}}\right] .
\end{aligned}
$$

Now, for $h:\left[0, T / p^{2}\right] \rightarrow \mathbb{R}$, we transform the linear second-order $(p, q)$-difference equation with separated boundary condition

$$
\begin{aligned}
D_{p, q}^{2} x(t) & =h(t), \quad t \in\left[0, \frac{T}{p^{2}}\right], \\
\alpha_{1} x(0)+\alpha_{2} D_{p, q} x(0) & =\alpha_{3}, \\
\beta_{1} x(T)+\beta_{2} D_{p, q} x\left(\frac{T}{p}\right) & =\beta_{3},
\end{aligned}
$$

into an integral equation which will be used to define the solution of the boundary value problem (5). For convenience we put constants $\Lambda=\alpha_{1}\left(\beta_{1} T+\beta_{2}\right)-\alpha_{2} \beta_{1} \neq 0$,

$$
\begin{aligned}
& \Lambda_{1}=\frac{\alpha_{3}\left(\beta_{1} T+\beta_{2}\right)-\alpha_{2} \beta_{3}}{\Lambda}, \\
& \Lambda_{2}=\frac{\alpha_{1} \beta_{3}-\alpha_{3} \beta_{1}}{\Lambda} .
\end{aligned}
$$

Lemma 4. The unique solution of linear problem (18) is given by

$$
\begin{aligned}
x(t)= & \Lambda_{1}+\Lambda_{2} t \\
& +\frac{\left(\alpha_{2}-\alpha_{1} t\right) \beta_{1}}{p \Lambda} \int_{0}^{T}(T-q s) h\left(\frac{s}{p}\right) d_{p, q} s \\
& +\frac{\left(\alpha_{2}-\alpha_{1} t\right) \beta_{2}}{\Lambda} \int_{0}^{T / p} h(s) d_{p, q} s \\
& +\frac{1}{p} \int_{0}^{t}(t-q s) h\left(\frac{s}{p}\right) d_{p, q} s .
\end{aligned}
$$

Proof. Applying $(p, q)$-integration to the first equation of (18), we have

$$
D_{p, q} x(t)-D_{p, q} x(0)=\int_{0}^{t} h(s) d_{p, q} s, \quad t \in\left[0, \frac{T}{p}\right] .
$$

Taking $(p, q)$-integration to (21) and using Theorem 3, we have

$$
\begin{aligned}
x(t)= & x(0)+D_{p, q} x(0) t \\
& +\frac{1}{p} \int_{0}^{t}(t-q s) h\left(\frac{s}{p}\right) d_{p, q} s, \quad t \in[0, T] .
\end{aligned}
$$

For convenience, we set constants $x(0)=A, D_{p, q} x(0)=B$ and then

$$
x(t)=A+B t+\frac{1}{p} \int_{0}^{t}(t-q s) h\left(\frac{s}{p}\right) d_{p, q} s .
$$

In particular for $t=T$ in (23), we have

$$
x(T)=A+B T+\frac{1}{p} \int_{0}^{T}(T-q s) h\left(\frac{s}{p}\right) d_{p, q} s .
$$

Using (21) and by replacing $t=T / p$, we obtain

$$
D_{p, q} x\left(\frac{T}{p}\right)=B+\int_{0}^{T / p} h(s) d_{p, q} s .
$$

From the first boundary condition of (18) and the above setting constants, we get

$$
\alpha_{1} A+\alpha_{2} B=\alpha_{3} .
$$

The second boundary condition of (18) with (24) and (25) yields

$$
\begin{gathered}
\beta_{1}\left(A+B T+\frac{1}{p} \int_{0}^{T}(T-q s) h\left(\frac{s}{p}\right) d_{p, q} s\right) \\
+\beta_{2}\left(B+\int_{0}^{T / p} h(s) d_{p, q} s\right)=\beta_{3} .
\end{gathered}
$$


Solving (26) and (27) for constants $A$ and $B$, it follows that

$$
\begin{aligned}
A & =\Lambda_{1}+\frac{1}{\Lambda}\left(\frac{\alpha_{2} \beta_{1}}{p} \int_{0}^{T}(T-q s) h\left(\frac{s}{p}\right) d_{p, q} s\right. \\
& \left.+\alpha_{2} \beta_{2} \int_{0}^{T / p} h(s) d_{p, q} s\right)
\end{aligned}
$$

and

$$
\begin{aligned}
B & =\Lambda_{2}-\frac{1}{\Lambda}\left(\frac{\alpha_{1} \beta_{1}}{p} \int_{0}^{T}(T-q s) h\left(\frac{s}{p}\right) d_{p, q} s\right. \\
& \left.+\alpha_{1} \beta_{2} \int_{0}^{T / p} h(s) d_{p, q} s\right) .
\end{aligned}
$$

Substituting constants $A$ and $B$ into (23), we obtain the unique solution in (20) of linear problem (18). The proof is completed.

\section{Main Results}

Let $\mathscr{C}:=C([0, T], \mathbb{R})$ denote the Banach space of all continuous functions from $[0, T]$ to $\mathbb{R}$ endowed with supnorm $\|x\|=\sup \{|x(t)|: t \in[0, T]\}$. In view of Lemma 4 we define an operator $\mathscr{A}: \mathscr{C} \longrightarrow \mathscr{C}$ by

$$
\begin{aligned}
& (\mathscr{A} x)(t) \\
& =\Lambda_{1}+\Lambda_{2} t \\
& \quad+\frac{\left(\alpha_{2}-\alpha_{1} t\right) \beta_{1}}{p \Lambda} \int_{0}^{T}(T-q s) f(s, x(p s)) d_{p, q} s \\
& \quad+\frac{\left(\alpha_{2}-\alpha_{1} t\right) \beta_{2}}{\Lambda} \int_{0}^{T / p} f\left(s, x\left(p^{2} s\right)\right) d_{p, q} s \\
& \quad+\frac{1}{p} \int_{0}^{t}(t-q s) f(s, x(p s)) d_{p, q} s .
\end{aligned}
$$

It should be noticed that problem (5) has solutions if and only if the operator $\mathscr{A}$ of equation $x=\mathscr{A} x$ has fixed points. Our first result is an existence theorem for separated boundary value problem of quantum $(p, q)$-difference equation (5) by using Schaefer's fixed point theorem.

Theorem 5. Assume that

$\left(H_{1}\right) f:\left[0, T / p^{2}\right] \times \mathbb{R} \rightarrow \mathbb{R}$ is a continuous function and there exists a constant $N>0$ such that

$$
\left|f\left(t, x\left(p^{2} t\right)\right)\right| \leq N
$$

for each $t \in\left[0, T / p^{2}\right]$ and all $x \in \mathbb{R}$.

Then the separated boundary value problem (5) has at least one solution on $[0, T]$.

Proof. Schaefer's fixed point theorem is used to prove that the operator $\mathscr{A}$ defined by (30) has at least one fixed point. So, we divide the proof into four steps.
Step 1 (continuity of $\mathscr{A}$ ). Let $\left\{x_{n}\right\}$ be a sequence of function such that $x_{n} \longrightarrow x$ on $\left[0, T / p^{2}\right]$. Given that $f$ is continuous function on $\left[0, T / p^{2}\right]$, we have

$$
f\left(t, x_{n}\left(p^{2} t\right)\right) \longrightarrow f\left(t, x\left(p^{2} t\right)\right)
$$

Therefore, we obtain

$$
\begin{aligned}
& \left|\left(\mathscr{A} x_{n}\right)(t)-(\mathscr{A} x(t))\right| \leq \frac{\left(\left|\alpha_{2}\right|+\left|\alpha_{1}\right| T\right)\left|\beta_{1}\right|}{p|\Lambda|} \\
& \cdot \int_{0}^{T}(T-q s)\left|f\left(s, x_{n}(p s)\right)-f(s, x(p s))\right| d_{p, q} s \\
& +\frac{\left(\left|\alpha_{2}\right|+\left|\alpha_{1}\right| T\right)\left|\beta_{2}\right|}{|\Lambda|} \\
& \cdot \int_{0}^{T / p}\left|f\left(s, x_{n}\left(p^{2} s\right)\right)-f\left(s, x\left(p^{2} s\right)\right)\right| d_{p, q} s+\frac{1}{p} \\
& \cdot \int_{0}^{t}(t-q s)\left|f\left(s, x_{n}(p s)\right)-f(s, x(p s))\right| d_{p, q} s,
\end{aligned}
$$

which implies that $\left\|\mathscr{A} x_{n}-\mathscr{A} x\right\| \longrightarrow 0$ as $n \longrightarrow \infty$. Hence the operator $\mathscr{A}$ is continuous.

Step $2(\mathscr{A}$ maps bounded sets into bounded sets in $[0, T])$. Choosing $r>0$, we define a bounded ball $B_{r}$ as $B_{r}=\{x \in \mathscr{C}$ : $\|x\| \leq r\}$. Then, for any $x \in B_{r}$, we have

$$
\begin{aligned}
& |(\mathscr{A} x)(t)| \leq\left|\Lambda_{1}\right|+\left|\Lambda_{2} t\right|+\frac{\left(\left|\alpha_{2}\right|+\left|\alpha_{1}\right| T\right)\left|\beta_{1}\right|}{p|\Lambda|} \\
& \cdot \int_{0}^{T}(T-q s)|f(s, x(p s))| d_{p, q} s \\
& +\frac{\left(\left|\alpha_{2}\right|+\left|\alpha_{1}\right| T\right)\left|\beta_{2}\right|}{|\Lambda|} \int_{0}^{T / p}\left|f\left(s, x\left(p^{2} s\right)\right)\right| d_{p, q} s \\
& +\frac{1}{p} \int_{0}^{t}(t-q s)|f(s, x(p s))| d_{p, q} s \leq\left|\Lambda_{1}\right|+\left|\Lambda_{2} T\right| \\
& +\frac{\left(\left|\alpha_{2}\right|+\left|\alpha_{1}\right| T\right)\left|\beta_{1}\right|}{p|\Lambda|} \int_{0}^{T}(T-q s) N d_{p, q} s \\
& +\frac{\left(\left|\alpha_{2}\right|+\left|\alpha_{1}\right| T\right)\left|\beta_{2}\right|}{|\Lambda|} \int_{0}^{T / p} N d_{p, q} s+\frac{1}{p} \\
& \cdot \int_{0}^{T}(T-q s) N d_{p, q} s=\left|\Lambda_{1}\right|+\left|\Lambda_{2} T\right| \\
& +\frac{\left(\left|\alpha_{2}\right|+\left|\alpha_{1}\right| T\right) T^{2}\left|\beta_{1}\right|}{(p+q)|\Lambda|} N+\frac{\left(\left|\alpha_{2}\right|+\left|\alpha_{1}\right| T\right) T\left|\beta_{2}\right|}{p|\Lambda|} \\
& N+\frac{\mathrm{T}^{2}}{p+q} N:=\rho
\end{aligned}
$$

This means that $\|\mathscr{A} x\| \leq \rho$. Therefore, the set $\mathscr{A} B_{r}$ is uniformly bounded. 
Step 3 ( $\mathscr{A}$ maps bounded sets into equicontinuous sets of $[0, T])$. Let $\tau_{1}, \tau_{2} \in[0, T]$ with $\tau_{1}<\tau_{2}$ be two points and $B_{r}$ be a bounded ball in $\mathscr{C}$. Then for any $x \in B_{r}$, we get

$$
\begin{aligned}
& \left|(\mathscr{A} x)\left(\tau_{2}\right)-(\mathscr{A} x)\left(\tau_{1}\right)\right| \leq\left(\tau_{2}-\tau_{1}\right)\left|\Lambda_{2}\right| \\
& +\frac{\left(\tau_{2}-\tau_{1}\right)\left|\alpha_{1} \beta_{1}\right|}{p|\Lambda|} \int_{0}^{T}(T-q s)|f(s, x(p s))| d_{p, q} s \\
& +\frac{\left(\tau_{2}-\tau_{1}\right)\left|\alpha_{1} \beta_{2}\right|}{|\Lambda|} \int_{0}^{T / p}\left|f\left(s, x_{n}\left(p^{2} s\right)\right)\right| d_{p, q} s \\
& +\frac{1}{p} \mid \int_{0}^{\tau_{2}}\left(\tau_{2}-q s\right) f(s, x(p s)) d_{p, q} s \\
& +\int_{0}^{\tau_{1}}\left(\tau_{1}-q s\right) f(s, x(p s)) d_{p, q} s\left|\leq\left(\tau_{2}-\tau_{1}\right)\right| \Lambda_{2} \mid \\
& +\frac{\left(\tau_{2}-\tau_{1}\right)\left|\alpha_{1} \beta_{1}\right|}{p|\Lambda|} \int_{0}^{T}(T-q s)|f(s, x(p s))| d_{p, q} s \\
& +\frac{\left(\tau_{2}-\tau_{1}\right)\left|\alpha_{1} \beta_{2}\right|}{|\Lambda|} \int_{0}^{T / p}\left|f\left(s, x_{n}\left(p^{2} s\right)\right)\right| d_{p, q} s \\
& +\frac{\left(\tau_{2}-\tau_{1}\right)\left|\alpha_{1} \beta_{2}\right|}{|\Lambda|} \int_{0}^{T / p}\left|f\left(s, x_{n}\left(p^{2} s\right)\right)\right| d_{p, q} s \\
& +\frac{1}{p} \mid \int_{0}^{\tau_{1}}\left(\tau_{2}-\tau_{1}\right) f(s, x(p s)) d_{p, q} s \\
& +\int_{\tau_{1}}^{\tau_{2}}\left(\tau_{2}-q s\right) f(s, x(p s)) d_{p, q} s\left|\leq\left(\tau_{2}-\tau_{1}\right)\right| \Lambda_{2} \mid \\
& +\tau_{1} \beta_{1}\left|d_{p, q}^{T}(T-q s)\right| f(s, x(p s)) \mid d_{p, q} s \\
& +\tau_{\tau_{1}} \\
& \left.\left.+\tau_{2}-q s\right) d_{p, q} s\right\}
\end{aligned}
$$

As $\tau_{1} \longrightarrow \tau_{2}$, the right-hand side of the above inequality (which is independent of $x$ ) tends to zero. This shows that the set $\mathscr{A} B_{r}$ is equicontinuous set. From a consequence of Steps 1 to 3, together with the Arzelá-Ascoli theorem, we deduce that the operator $\mathscr{A}: \mathscr{C} \longrightarrow \mathscr{C}$ is completely continuous.

Step 4. Finally, we show that the set

$$
E=\{x \in \mathscr{C}: x=\theta \mathscr{A} x \text { for some } 0<\theta<1\}
$$

is bounded.

Let $x \in E$ be a solution of problem (5). Then $x(t)=$ $\theta(\mathscr{A} x)(t)$ for some $0<\theta<1$. Hence, for each $t \in[0, T]$, by the method of computation in Step 2, we obtain

$$
\|\mathscr{A} x\| \leq \rho .
$$

This gives that the set $E$ is bounded. By applying Schaefer's fixed point theorem, we get that $\mathscr{A}$ has at least one fixed point which is a solution of the second-order quantum $(p, q)$ difference equation with separated boundary value problem (5) on $[0, T]$. The proof is completed.
The second existence of a unique solution is based on Banach's contraction mapping principle. For convenience we set a positive constant

$$
\Phi=\frac{\left(\left|\alpha_{2}\right|+\left|\alpha_{1}\right| T\right) T}{|\Lambda|}\left\{\frac{\left|\beta_{1}\right| T}{p+q}+\frac{\left|\beta_{2}\right|}{p}\right\}+\frac{T^{2}}{p+q} .
$$

Theorem 6. Assume that the function $f:\left[0, T / p^{2}\right] \times \mathbb{R} \rightarrow \mathbb{R}$ is continuous and satisfying the assumption:

$\left(\mathrm{H}_{2}\right)$ There exists constant $L>0$ such that $\mid f(t, x)-$ $f(t, y)|\leq L| x-y \mid$ for each $t \in\left[0, T / p^{2}\right]$ and $x, y \in \mathbb{R}$. If

$$
L \Phi<1
$$

where $\Phi$ is defined by (38), then the boundary value problem of quantum $(p, q)$-difference equation (5) has a unique solution on $[0, T]$.

Proof. The existence of a unique fixed point of operator equation $x=\mathscr{A} x$, where the operator $\mathscr{A}$ is defined by (30), is proved by using Banach's contraction mapping principle. Let us define a ball $B_{r}=\{x \in \mathscr{C}:\|x\| \leq r\}$ with the value $r$ satisfying

$$
r \geq \frac{\left|\Lambda_{1}\right|+\left|\Lambda_{2} T\right|+M \Phi}{1-L \Phi}
$$

where $M=\sup _{t \in\left[0, T / p^{2}\right]}|f(t, 0)|$. Note that the above inequality is well defined as $L \Phi<1$. Next, we will show that $\mathscr{A} B_{r} \subset B_{r}$. For any $x \in B_{r}$, we have

$$
\begin{aligned}
& |(\mathscr{A} x)(t)| \leq \sup _{t \in\left[0, T / p^{2}\right]}\left\{\left|\Lambda_{1}\right|+\left|\Lambda_{2}\right| t+\frac{\left|\left(\alpha_{2}-\alpha_{1} t\right) \beta_{1}\right|}{p|\Lambda|}\right. \\
& \cdot \int_{0}^{T}(T-q s)|f(s, x(p s))| d_{p, q} s \\
& +\frac{\left|\left(\alpha_{2}-\alpha_{1} t\right) \beta_{2}\right|}{|\Lambda|} \int_{0}^{T / p}\left|f\left(s, x_{n}\left(p^{2} s\right)\right)\right| d_{p, q} s+\frac{1}{p} \\
& \left.\cdot \int_{0}^{T}(T-q s)|f(s, x(p s))| d_{p, q} s\right\} \leq\left|\Lambda_{1}\right| \\
& +\left|\Lambda_{2}\right| T+\frac{\left(\left|\alpha_{2}\right|+\left|\alpha_{1}\right| T\right)\left|\beta_{1}\right|}{|p \Lambda|} \int_{0}^{T}(T-q s) \\
& \cdot(|f(s, x(p s))-f(s, 0)|+|f(s, 0)|) d_{p, q} s \\
& +\frac{\left(\left|\alpha_{2}\right|+\left|\alpha_{1}\right| T\right)\left|\beta_{2}\right|}{|\Lambda|} \\
& +|f(s, 0)|) d_{p, q} s+\frac{1}{p} \int_{0}^{T}(T-q s) \\
& +\int_{0}^{T / p}\left(\left|f\left(s, x\left(p^{2} s\right)\right)-f(s, 0)\right|\right.
\end{aligned}
$$




$$
\begin{aligned}
& \leq\left|\Lambda_{1}\right|+\left|\Lambda_{2}\right| T+(L\|x\|+M) \\
& \cdot\left\{\frac{\left(\left|\alpha_{2}\right|+\left|\alpha_{1}\right| T\right)\left|\beta_{1}\right|}{p|\Lambda|} \int_{0}^{T}(T-q s) d_{p, q} s\right. \\
& +\frac{\left(\left|\alpha_{2}\right|+\left|\alpha_{1}\right| T\right)\left|\beta_{2}\right|}{|\Lambda|} \int_{0}^{T / p} d_{p, q} s+\frac{1}{p} \\
& \left.\cdot \int_{0}^{T}(T-q s) d_{p, q} s\right\} \leq\left|\Lambda_{1}\right|+\left|\Lambda_{2}\right| T+(L r \\
& +M)\left\{\frac{\left(\left|\alpha_{2}\right|+\left|\alpha_{1}\right| T\right) T^{2}\left|\beta_{1}\right|}{(p+q)|\Lambda|}\right. \\
& \left.+\frac{\left(\left|\alpha_{2}\right|+\left|\alpha_{1}\right| T\right) T\left|\beta_{2}\right|}{p|\Lambda|}+\frac{T^{2}}{p+q}\right\}=\left|\Lambda_{1}\right|+\left|\Lambda_{2}\right| T \\
& +(L r+M) \Phi \leq r,
\end{aligned}
$$

which implies that $\|\mathscr{A} x\|<r$. Therefore, we have $\mathscr{A} B_{r} \subset B_{r}$. Finally we shall show that $\mathscr{A}$ is a contraction. For any $x, y \in \mathscr{C}$ and for each $t \in[0, T]$, we obtain

$$
\begin{aligned}
& |\mathscr{A} x(t)-\mathscr{A} y(t)| \leq \frac{\left(\left|\alpha_{2}\right|+\left|\alpha_{1}\right| T\right)\left|\beta_{1}\right|}{|p \Lambda|} \int_{0}^{T}(T-q s) \\
& \cdot(|f(s, x(p s))-f(s, y(p s))|) d_{p, q} s \\
& +\frac{\left(\left|\alpha_{2}\right|+\left|\alpha_{1}\right| T\right)\left|\beta_{2}\right|}{|\Lambda|} \int_{0}^{T / p}\left(\mid f\left(s, x\left(p^{2} s\right)\right)\right. \\
& \left.-f\left(s, y\left(p^{2} s\right)\right) \mid\right) d_{p, q} s+\frac{1}{p} \int_{0}^{T}(T-q s) \\
& \cdot(|f(s, x(p s))-f(s, x(p s))|) d_{p, q} s \\
& \leq\|x-y\|\left\{\frac{\left(\left|\alpha_{2}\right|+\left|\alpha_{1}\right| T\right) T^{2}\left|\beta_{1}\right|}{(p+q)|\Lambda|}\right. \\
& \left.+\frac{\left(\left|\alpha_{2}\right|+\left|\alpha_{1}\right| T\right) T\left|\beta_{2}\right|}{p|\Lambda|}+\frac{T^{2}}{p+q}\right\}=L \Phi\|x-y\| .
\end{aligned}
$$

Therefore, we have $\|\mathscr{A} x-\mathscr{A} y\| \leq L \Phi\|x-y\|$. As $L \Phi<1$, then the operator $\mathscr{A}$ is a contraction. Hence, by the Banach contraction mapping principle, it follows that the operator $\mathscr{A}$ has a unique fixed point which is the unique solution of problem (5) on $[0, T]$. This completes the proof.

The third existence theorem is based on Lelay-Schauder's nonlinear alternative.

Lemma 7 (nonlinear alternative for single-value maps [43]). Let $E$ be a Banach space, $C$ be a closed, convex subset of $E, U$ be an open subset of $C$, and $0 \in U$. Suppose that $A: \bar{U} \longrightarrow C$ is continuous and compact (that is, $A(\bar{U})$ is a relatively compact subset of $C$ map). Then either

(i) A has a fixed point in $\bar{U}$, or (ii) there is a $x \in \partial U$ (the boundary of $U$ in $C$ ) and $\lambda \epsilon$ $(0,1)$ with $x=\lambda A(x)$.

Theorem 8. Assume that $f:\left[0, T / p^{2}\right] \times \mathbb{R} \longrightarrow \mathbb{R}$ is a continuous function. In addition we suppose that

$\left(H_{3}\right)$ there exist a continuous nondecreasing function $\psi:[0, \infty) \longrightarrow(0, \infty)$ and a function $z \in C\left(\left[0, T / p^{2}\right], \mathbb{R}^{+}\right)$such that

$$
\begin{aligned}
& |f(t, x)| \leq z(t) \psi(\|x\|) \\
& \qquad \text { for each }(t, x) \in\left[0, \frac{T}{p^{2}}\right] \times \mathbb{R} ;
\end{aligned}
$$

$\left(H_{4}\right)$ there exists a constant $K>0$ such that

$$
\frac{K}{\left|\Lambda_{1}\right|+\left|\Lambda_{2}\right| T+\|z\| \psi(K) \Phi}>1 .
$$

Then the separated boundary value problem (5) has at least one solution on $[0, T]$.

Proof. As in the proof of Theorem 5, the operator $\mathscr{A}: \mathscr{C} \longrightarrow$ $\mathscr{C}$ is completely continuous. The result will follow from the Lelay-Schauder nonlinear alternative (Lemma 7) once we have proved the boundedness of the set of all solutions to equations $x=\theta \mathscr{A} x$ for $\theta \in[0,1]$.

Let $x$ be a solution of problem (5). Then, from $\left(\mathrm{H}_{3}\right)$, we have

$$
\begin{aligned}
\|x\| & \leq\left|\Lambda_{1}\right|+\left|\Lambda_{2} T\right|+\|z\| \psi(\|x\|) \\
& \left\{\frac{\left(\left|\alpha_{2}\right|+\left|\alpha_{1}\right| T\right) T^{2}\left|\beta_{1}\right|}{(p+q)|\Lambda|}+\frac{\left(\left|\alpha_{2}\right|+\left|\alpha_{1}\right| T\right) T\left|\beta_{2}\right|}{p|\Lambda|}\right. \\
& \left.+\frac{T^{2}}{p+q}\right\}=\left|\Lambda_{1}\right|+\left|\Lambda_{2} T\right|+\|z\| \psi(\|x\|) \Phi .
\end{aligned}
$$

Thus, we obtain

$$
\frac{\|x\|}{\left|\Lambda_{1}\right|+\left|\Lambda_{2}\right| T+\|z\| \psi(\|x\|) \Phi} \leq 1 .
$$

In view of $\left(H_{4}\right)$, there exists a positive constant $K$ such that $\|x\| \neq K$. Let us define the set

$$
U=\{x \in \mathscr{C}:\|x\|<K\} .
$$

Note that the operator $\mathscr{A}: \bar{U} \longrightarrow \mathscr{C}$ is continuous and completely continuous. From the choice of $U$, there is no $x \in \partial U$ such that $x=\theta \mathscr{A} x$ for some $\theta \in(0,1)$. Therefore, by applying the nonlinear alternative of LeraySchauder type, we can conclude that the operator $\mathscr{A}$ has at least one fixed point in $\bar{U}$, which is a solution of the quantum $(p, q)$-difference boundary value problem $(5)$ on $[0, T]$. The proof is completed.

The final existence theorem is established by using Krasoselskii's fixed point theorem. 
Lemma 9 (Krasnoselskii's fixed point theorem [44]). Let $M$ be a closed, bounded, convex, and nonemtry subset of a Banach space $X$. Let $A, B$ be the operators such that (a) $A x+B y \in M$ whenever $x, y \in M$; $(b) A$ is compact and continuous; (c) $B$ is a contraction mapping. Then there exists $z \in M$ such that $z=A z+B z$.

Theorem 10. Assume that $f:\left[0, T / p^{2}\right] \times \mathbb{R} \rightarrow \mathbb{R}$ is a continuous function satisfying the assumption $\left(\mathrm{H}_{2}\right)$. In addition we suppose that

$\left(H_{5}\right)|f(t, x)| \leq \kappa(t), \forall(t, x) \in\left[0, T / p^{2}\right] \times \mathbb{R}$ and $\kappa \in$ $C\left(\left[0, T / p^{2}\right], \mathbb{R}^{+}\right)$.

If

$$
\left(\frac{T^{2}}{p+q}\right) L<1
$$

then the separated boundary value problem of quantum $(p, q)$ difference equation (5) has at least one solution on $[0, T]$.

Proof. Let us define $\sup _{t \in\left[0, T / p^{2}\right]}|\kappa(t)|=\|\kappa\|$ and choose a suitable constant $\bar{r}$ as

$$
\bar{r} \geq\left|\Lambda_{1}\right|+\left|\Lambda_{2}\right| T+\|\kappa\| \Phi
$$

where $\Phi$ is defined by (38). Furthermore, we define the operators $\mathscr{P}$ and $\mathcal{Q}$ on $B_{\bar{r}}=\{x \in \mathscr{C}:\|x\| \leq \bar{r}\}$ by

$(\mathscr{P} x)(t)$

$$
\begin{gathered}
=\frac{\left(\alpha_{2}-\alpha_{1} t\right) \beta_{1}}{p \Lambda} \int_{0}^{T}(T-q s) f(s, x(p s)) d_{p, q} s \\
+\frac{\left(\alpha_{2}-\alpha_{1} t\right) \beta_{2}}{\Lambda} \int_{0}^{T / p} f\left(s, x\left(p^{2} s\right)\right) d_{p, q} s,
\end{gathered}
$$

$(Q y)(t)$

$$
=\Lambda_{1}+\Lambda_{2} t+\frac{1}{p} \int_{0}^{t}(t-q s) f(s, y(p s)) d_{p, q} s .
$$

Observe that $\mathscr{P} x+\mathbb{Q} x=\mathscr{A} x$. For $x, y \in B_{\bar{r}}$, we have

$$
\begin{aligned}
& \|\mathscr{P} x+\mathscr{Q} y\| \leq\left|\Lambda_{1}\right|+\left|\Lambda_{2} T\right|+\|\kappa\| \\
& \quad \cdot\left\{\frac{\left(\left|\alpha_{2}\right|+\left|\alpha_{1}\right| T\right) T^{2}\left|\beta_{1}\right|}{(p+q)|\Lambda|}+\frac{\left(\left|\alpha_{2}\right|+\left|\alpha_{1}\right| T\right) T\left|\beta_{2}\right|}{p|\Lambda|}\right. \\
& \left.+\frac{T^{2}}{p+q}\right\}=\left|\Lambda_{1}\right|+\left|\Lambda_{2} T\right|+\|\kappa\| \Phi \leq \bar{r},
\end{aligned}
$$

which yields $\mathscr{P} x+Q \mathcal{Q} y \in B_{\bar{r}}$. Therefore, the condition (a) of Lemma 9 is satisfied. It follows from the assumption $\left(\mathrm{H}_{2}\right)$ together with (48) that $Q$ is a contraction mapping. Hence the condition $(c)$ of Lemma 9 is fulfilled. Now we will show that the condition $(b)$ of Lemma 9 is satisfied. Given that function $f$ is continuous, we get that the operator $\mathscr{P}$ is continuous. It is easy to verify that

$$
\begin{gathered}
\|\mathscr{P} x\| \leq\|\kappa\|\left\{\frac{\left(\left|\alpha_{2}\right|+\left|\alpha_{1}\right| T\right) T^{2}\left|\beta_{1}\right|}{(p+q)|\Lambda|}\right. \\
\left.+\frac{\left(\left|\alpha_{2}\right|+\left|\alpha_{1}\right| T\right) T\left|\beta_{2}\right|}{p|\Lambda|}\right\} .
\end{gathered}
$$

Thus, the set $\mathscr{P}\left(B_{r}\right)$ is uniformly bounded. Next we prove the compactness of the operator $\mathscr{P}$. Give $\sup _{(t, x) \in\left[0, T / p^{2}\right] \times B_{\bar{r}}}|f(t, x)|=\bar{f}<\infty$ and let $t_{1}, t_{2} \in[0, T]$ with $t_{1}<t_{2}$. Then we get

$$
\begin{aligned}
& \left|(\mathscr{P} x)\left(t_{2}\right)-\left(\mathscr{P} x\left(t_{1}\right)\right)\right| \\
& \leq \frac{\left|\alpha_{1} \beta_{1}\right|\left(t_{2}-t_{1}\right)}{p|\Lambda|} \int_{0}^{T}(T-q s)|f(s, x(p s))| d_{p, q} s \\
& \quad+\frac{\left|\alpha_{1} \beta_{2}\right|\left(t_{2}-t_{1}\right)}{|\Lambda|} \int_{0}^{T / p}\left|f\left(s, x\left(p^{2} s\right)\right)\right| d_{p, q} s \\
& \leq \bar{f}\left\{\frac{\left|\alpha_{1} \beta_{1}\right| T^{2}}{(p+q)|\Lambda|}+\frac{\left|\alpha_{1} \beta_{2}\right| T}{|\Lambda|}\right\}\left(t_{2}-t_{1}\right),
\end{aligned}
$$

which is independent of $x$ and tends to zero as $t_{1} \longrightarrow t_{2}$. So, the set $\mathscr{P}\left(B_{r}\right)$ is equicontinuous. By the Arzelá-Ascoli theorem, $\mathscr{P}$ is compact on $B_{\bar{r}}$. Thus all the assumptions of Lemma 9 are satisfied. Therefore the separated boundary value problem of quantum $(p, q)$-difference equation (5) has at least one solution on $[0, T]$. The proof is completed.

\section{Examples}

Example 1. Consider the following separated boundary value problem for second-order quantum $(p, q)$-difference equation of the form

$$
D_{1 / 2,1 / 5}^{2} x(t)=f\left(t, x\left(\frac{t}{4}\right)\right), \quad t \in[0,4],
$$

$$
\begin{aligned}
& \frac{5}{6} x(0)+\frac{4}{5} D_{p, q} x(0)=\frac{1}{3} \\
& \frac{1}{4} x(1)+\frac{5}{6} D_{p, q} x(2)=\frac{3}{5} .
\end{aligned}
$$

Set constants $p=1 / 2, q=1 / 5, T=1, \alpha_{1}=5 / 6, \alpha_{2}=4 / 5$, $\alpha_{3}=1 / 3, \beta_{1}=1 / 4, \beta_{2}=5 / 6$, and $\beta_{3}=3 / 5$. Then we can find constants as $\Lambda=0.7027777778, \Lambda_{1}=-0.1691699605$, $\Lambda_{2}=0.5928853755$, and $\Phi=6.132128741$.

(i) Let $f:[0,4] \times \mathbb{R} \longrightarrow \mathbb{R}$ by

$$
f\left(t, x\left(\frac{t}{4}\right)\right)=\frac{5 t^{2}}{\left(1+x^{2}(t / 4)\right)}+\frac{t^{2} \sin t}{3 t+1} .
$$

It is easy to see that $|f(t, x)| \leq 5.25=N$ which satisfies $\left(H_{1}\right)$. Thus, by Theorem 5 , the separated boundary value problem (54) with $f$ given by $(55)$ has at least one solution on $[0,1]$.

(ii) Given $f:[0,4] \times \mathbb{R} \longrightarrow \mathbb{R}$ with

$$
f\left(t, x\left(\frac{t}{4}\right)\right)=\frac{|\cos t|}{18}\left(\frac{x^{2}(t / 4)+2|x(t / 4)|}{1+|x(t / 4)|}\right) .
$$


it follows that

$$
|f(t, x)-f(t, y)| \leq \frac{1}{9}|x-y| .
$$

Therefore, the condition $\left(H_{2}\right)$ is satisfied with $L=1 / 9$. Indeed, we have $L \Phi=0.6813476379<1$. Hence, by Theorem 6 , the separated boundary value problem of quantum $(p, q)$-difference equation (54) has a unique solution on $[0,1]$.

Example 2. Consider the following separated boundary value problem for second-order quantum $(p, q)$-difference equation of the form

$$
\begin{aligned}
& D_{1 / 3,1 / 6}^{2} x(t)=g\left(t, x\left(\frac{t}{9}\right)\right), \\
& t \in\left[0, \frac{9}{2}\right], \\
& \frac{7}{3} x(0)+\frac{5}{6} D_{p, q} x(0)=\frac{4}{7}, \\
& \frac{2}{5} x\left(\frac{1}{2}\right)+\frac{7}{2} D_{p, q} x\left(\frac{3}{2}\right)=\frac{6}{5} .
\end{aligned}
$$

Set constants $p=1 / 3, q=1 / 6, T=1 / 2, \alpha_{1}=7 / 3, \alpha_{2}=$ $5 / 6, \alpha_{3}=4 / 7, \beta_{1}=2 / 5, \beta_{2}=7 / 2$, and $\beta_{3}=6 / 5$. Then we can compute that $\Lambda=8.3, \Lambda_{1}=0.1342512908, \Lambda_{2}=$ 0.3098106712 , and $\Phi=1.813253012$.

(i) Let $g:[0,9 / 2] \times \mathbb{R} \longrightarrow \mathbb{R}$ be a nonlinear function defined by

$$
g\left(t, x\left(\frac{t}{9}\right)\right)=\frac{1}{(t+2)^{3}}\left(\frac{x^{4}(t / 9)}{1+x^{2}(t / 9)}+2\right) .
$$

Choosing $z(t)=1 /(t+2)^{3}$ and $\psi(x)=x^{2}+2$, we notice that the condition $\left(\mathrm{H}_{3}\right)$ of Theorem 8 is satisfied. Furthermore, we can find that $\|z\|=1 / 8$. Then there exists a constant $K$ such that $K \in(0.9447899357,3.467170197)$. Therefore, the separated boundary value problem (58) with $g$ given by (59) has at least one solution on $[0,1 / 2]$.

(ii) Let a function $g:[0,9 / 2] \times \mathbb{R} \rightarrow \mathbb{R}$ be defined by

$$
g\left(t, x\left(\frac{t}{9}\right)\right)=\left(\frac{3 e^{-t}}{2+t^{2}} \cdot \frac{|x(t / 9)|}{|x(t / 9)|+1}\right)+\frac{t}{2} .
$$

It follows that

$$
|g(t, x)| \leq \frac{3 e^{-t}}{2+t^{2}}+\frac{t}{2}
$$

Then the function $g$ defined in $(60)$ satisfies condition $\left(H_{5}\right)$ with $\kappa(t)=\left(\left(3 e^{-t}\right) /\left(2+t^{2}\right)\right)+(t / 2)$. In addition, the condition $\left(H_{2}\right)$ is satisfied with $L=3 / 2$ and

$$
\left(\frac{T^{2}}{p+q}\right) L=0.75<1 .
$$

Hence, by applying Theorem 10, the separated boundary value problem for second-order quantum $(p, q)$-difference equation (58) with $g$ defined in (60) has at least one solution on $[0,1 / 2]$.

Remark 3. In Example 2, Case (ii), although the function $g(t, x)$ satisfies condition $\left(H_{2}\right)$, Theorem 6 cannot be applied, since $L \Phi=2.719879518>1$.

\section{Data Availability}

No data were used to support this study.

\section{Conflicts of Interest}

The authors declare that there are no conflicts of interest regarding the publication of this paper.

\section{Acknowledgments}

This research was funded by King Mongkut's University of Technology North Bangkok, Contract no. KMUTNB-60ART-074.

\section{References}

[1] F. H. Jackson, “On q-difference integrals," The Quarterly Journal of Pure and Applied Mathematics, vol. 41, pp. 193-203, 1910.

[2] F. H. Jackson, " $q$-difference equations," American Journal of Mathematics, vol. 32, no. 4, pp. 305-314, 1910.

[3] R. D. Carmichael, "The general theory of Linear q-difference equations," The American Journal of Mathematics, vol. 34, no. 2, pp. 147-168, 1912.

[4] V. Kac and P. Cheung, Quantum Calculus, Springer, New York, NY, USA, 2002.

[5] T. Mason, "On properties of the solutions of linear $q$-difference equations with entire function coefficient," The American Journal of Mathematics, vol. 37, no. 4, pp. 439-444, 1915.

[6] C. R. Adams, "On the linear ordinary q-difference equation," Annals of Mathematics: Second Series, vol. 30, no. 1-4, pp. 195205,1928

[7] T. Ernst, The history of q-calculus and a new method, UUDMReport2000:16, Department of Mathematics, UppsalaUniversity, 2000.

[8] B. Ahmad and S. K. Ntouyas, "Boundary Value Problems for $q$ Difference Inclusions," Abstract and Applied Analysis, vol. 2011, Article ID 292860, 15 pages, 2011.

[9] A. Dobrogowska and A. Odzijewicz, "Second order $q$-difference equations solvable by factorization method," Journal of Computational and Applied Mathematics, vol. 193, no. 1, pp. 319-346, 2006.

[10] M. El-Shahed and H. A. Hassan, "Positive solutions of $q$ difference equation," Proceedings of the American Mathematical Society, vol. 138, no. 5, pp. 1733-1738, 2010.

[11] R. A. C. Ferreira, "Nontrivial solutions for fractional $q$ difference boundary value problems," Electronic Journal of Qualitative Theory of Differential Equations, vol. 70, pp. 1-10, 2010.

[12] G. Gasper and M. Rahman, Basic Hypergeometric Series, vol. 35 of Encyclopedia of Mathematics and its Applications, Cambridge University Press, Cambridge, UK, 1990.

[13] J. Ma and J. Yang, "Existence of solutions for multi-point boundary value problem of fractional q-difference equation," Electronic Journal of Qualitative Theory of Differential Equations, no. 92, pp. 1-10, 2011.

[14] J. Tariboon and S. K. Ntouyas, "Quantum calculus on finite intervals and applications to impulsive difference equations," Advances in Difference Equations, vol. 2013, article no. 282, 2013. 
[15] B. Ahmad, S. K. Ntouyas, and J. Tariboon, Quantum Calculus: New Concepts, Impulsive IVPs and BVPs, Inequalities, vol. 4 of Trends in Abstract and Applied Analysis, World Scientific Publishing, Hackensack, NJ, USA, 2016.

[16] R. J. Finkelstein, "Symmetry group of the hydrogen atom," Journal of Mathematical Physics, vol. 8, no. 3, pp. 443-449, 1967.

[17] R. J. Finkelstein, " $q$-field theory," Letters in Mathematical Physics, vol. 34, no. 2, pp. 169-176, 1995.

[18] F.-L. Chan and R. J. Finkelstein, " $q$-deformation of the Coulomb problem," Journal of Mathematical Physics, vol. 35, no. 7, pp. 3273-3284, 1994.

[19] A. C. Cadavid and R. J. Finkelstein, "The q-Coulomb problem in configuration space," Journal of Mathematical Physics, vol. 37, no. 8, pp. 3675-3683, 1996.

[20] R. J. Finkelstein, “The q-Coulomb problem," Journal of Mathematical Physics, vol. 37, no. 6, pp. 2628-2636, 1996.

[21] J. Feigenbaum and P. G. Freund, "A q-deformation of the Coulomb problem," Journal of Mathematical Physics, vol. 37, no. 4, pp. 1602-1616, 1996.

[22] H. Cheng, Canonical Quantization of Yang-Mills Theories, Perspectives in Mathematical Physics, International Press, 1996.

[23] R. J. Finkelstein, “q-gravity," Letters in Mathematical Physics, vol. 38, no. 1, pp. 53-62, 1996.

[24] M. Kamata and A. Nakamula, "One-parameter family of selfdual solutions in classical Yang-Mills theory," Physics Letters B: Particle Physics, Nuclear Physics and Cosmology, vol. 463, no. 24, pp. 257-262, 1999.

[25] T. Negadi and M. Kibler, "A q-deformed Aufbau Prinzip," Journal of Physics A: Mathematical and General, vol. 25, no. 4, pp. L157-L160, 1992.

[26] L. P. Marinova, P. P. Raychev, and J. Maruani, "Molecular backbending in AgH and its description in terms of q-algebras," Molecular Physics. An International Journal in the Field of Chemical Physics, vol. 82, no. 6, pp. 1115-1129, 1994.

[27] M. R-Monteiro, L. M. C. S. Rodrigues, and S. Wulck, "Quantum algebraic nature of the phonon spectrum in ${ }^{4} \mathrm{He}, "$ Physical Review Letters, vol. 76, no. 7, pp. 1098-1100, 1996.

[28] A. M. Gavrilik, "q-Serre relations in $\mathrm{U}_{q}\left(\mathrm{u}_{n}\right)$ and q-deformed meson mass sum rules," Journal of Physics A: Mathematical and General, vol. 27, no. 3, pp. L91-L94, 1994.

[29] R. J. Finkelstein, "q gauge theory," International Journal of Modern Physics A, vol. 11, no. 4, pp. 733-746, 1996.

[30] G. Kaniadakis, A. Lavagno, and P. Quarati, "Kinetic model for q-deformed bosons and fermions," Physics Letters A, vol. 227, no. 3-4, pp. 227-231, 1997.

[31] K. N. Ilinski, G. V. Kalinin, and A. S. Stepanenko, "q-functional field theory for particles with exotic statistics," Physics Letters A, vol. 232, no. 6, pp. 399-408, 1997.

[32] W. Siegel, Introduction to String Field Theory, vol. 8 of Advanced Series in Mathematical Physics, World Scientific Publishing, Teaneck, NJ, USA, 1988.

[33] R. Chakrabarti and R. Jagannathan, "A (p,q)-oscillator realization of two-parameter quantum algebras," Journal of Physics A: Mathematical and General, vol. 24, no. 24, pp. 5683-5701, 1991.

[34] R. Jagannathan and K. Srinivasa Rao, “Two-parameter quantum algebras, twin-basic number, and associated generalized hypergeometric series," Differential Equations \& Applications, vol. 27, 2006.

[35] V. Sahai and S. Yadav, "Representations of two parameter quantum algebras and p,q-special functions," Journal of Mathematical Analysis and Applications, vol. 335, no. 1, pp. 268-279, 2007.
[36] I. Burban, "Two-parameter deformation of the oscillator algebra and (p,q)-analog of two-dimensional conformal field theory," Journal of Nonlinear Mathematical Physics, vol. 2, no. 3-4, pp. 384-391, 1995.

[37] P. N. Sadjang, "On the fundamental theorem of (p, q)-calculus and some (p, q)-Taylor formulas," https://arxiv.org/abs/ 1309.3934, 2015.

[38] M. N. Hounkonnou and J. D. Kyemba, "R(p,q)-calculus: differentiation and integration," SUT Journal of Mathematics, vol. 49, no. 2, pp. 145-167, 2013.

[39] M. Mursaleen, K. J. Ansari, and A. Khan, "On (p,q)-analogue of Bernstein operators," Applied Mathematics and Computation, vol. 266, pp. 874-882, 2015.

[40] S. Araci, U. G. Duran, M. Acikgoz, and H. M. Srivastava, "A certain (p,q)-derivative operator and associated divided differences," Journal of Inequalities and Applications, vol. 2016, article no. 301, 2016.

[41] U. Duran, Post Quantum Calculus, [Master Thesis], University of Gaziantep, 2016.

[42] N. Kamsrisuk, C. Promsakon, S. K. Ntouyas, and J. Tariboon, "Nonlocal boundary value problems for (p,q)-difference equations," Differential Equations \& Applications, vol. 10, no. 2, pp. 183-195, 2018.

[43] A. Granas and J. Dugundji, Fixed Point Theory and Applications, Springer, New York, NY, USA, 2003.

[44] M. A. Krasnosel'skii, "Two remarks on the method of successive approximations," Uspekhi Matematicheskikh Nauk, vol. 10, no. 1, pp. 123-127, 1955. 


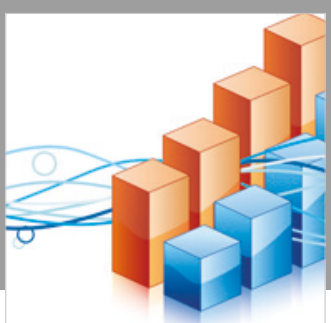

Advances in

Operations Research

\section{-n-m}
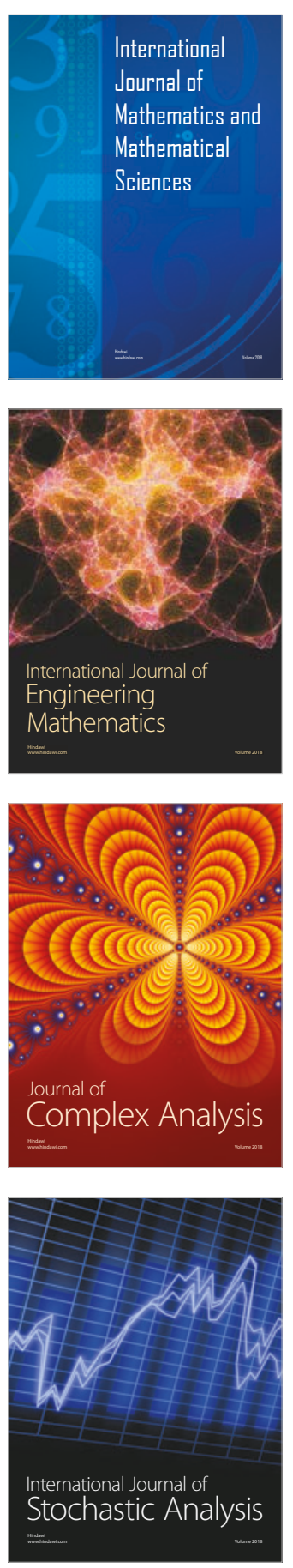
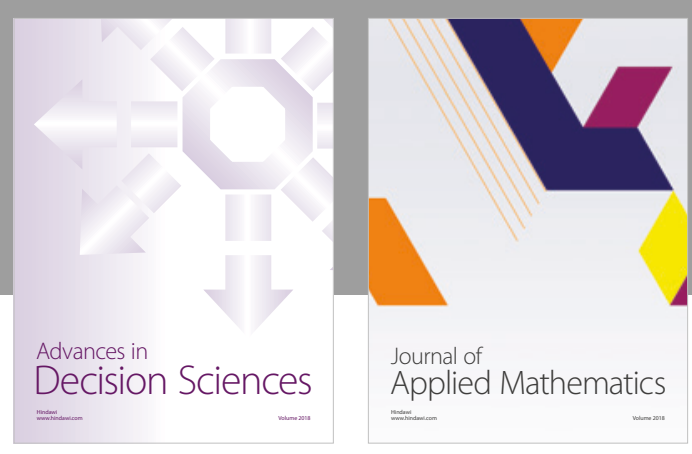

Journal of

Applied Mathematics
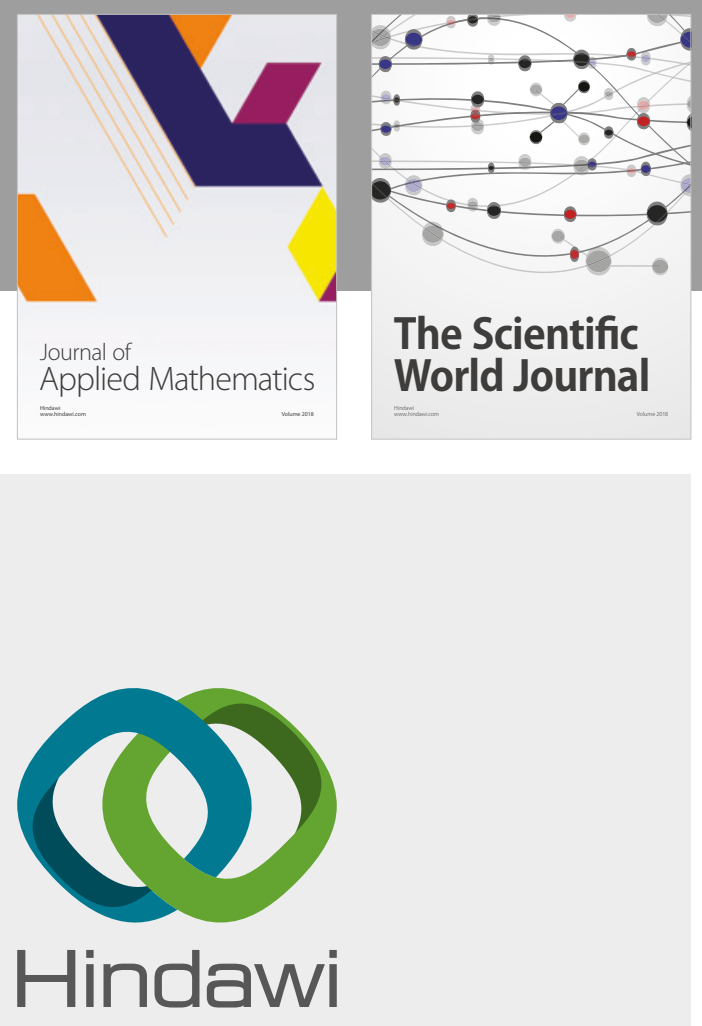

Submit your manuscripts at

www.hindawi.com

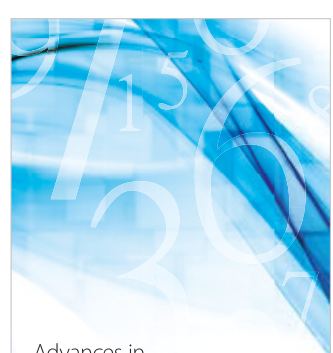

Advances in
Numerical Analysis
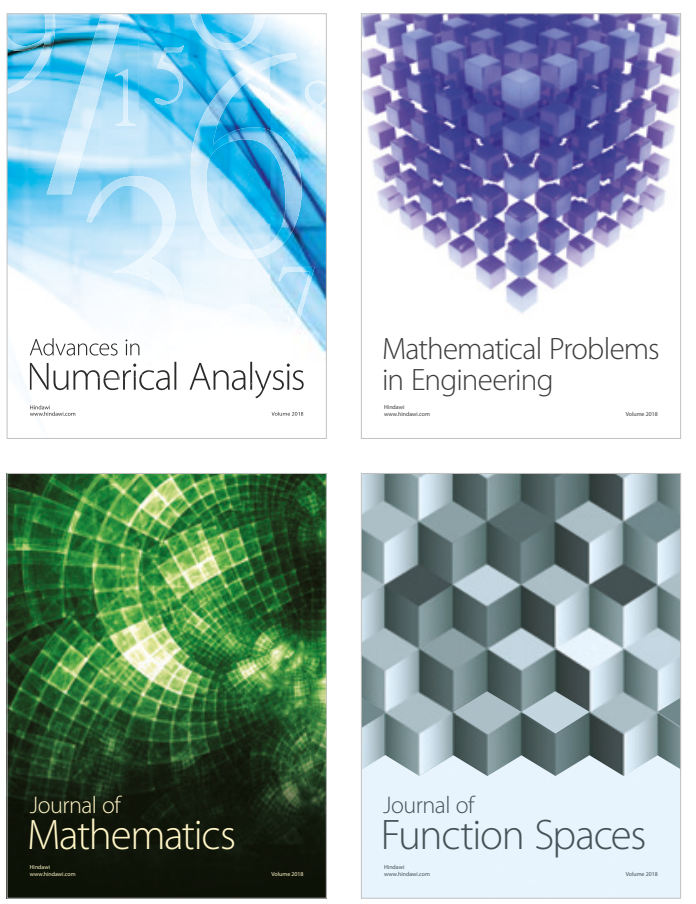

Mathematical Problems in Engineering

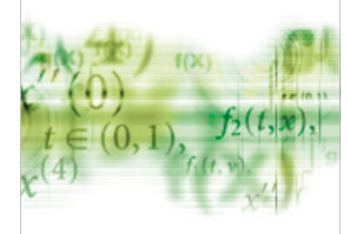

International Journal of

Differential Equations

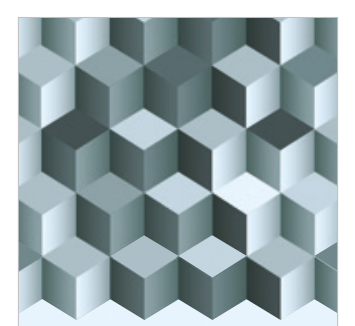

Journal of

Function Spaces

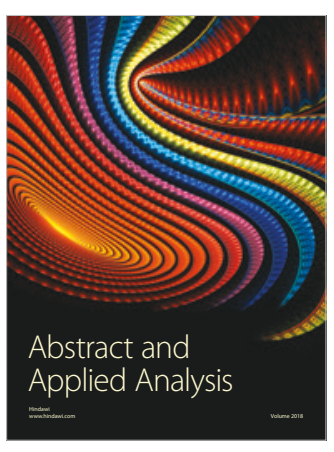

The Scientific

World Journal

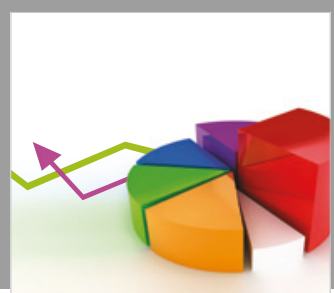

Journal of

Probability and Statistics
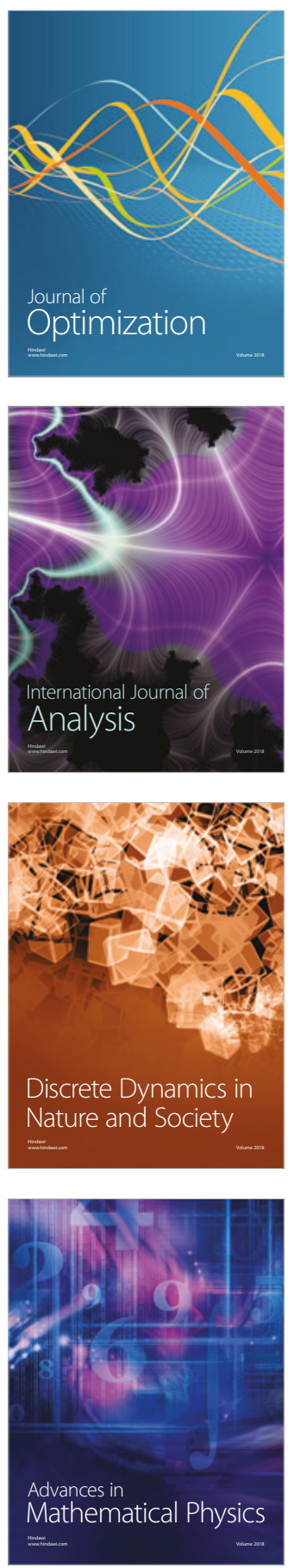